\title{
A General Creation-Annihilation Model with Absorbing States
}

\author{
Wellington G. Dantas, Armando Ticona, ${ }^{*}$ and Jürgen F. Stilck \\ Instituto de Física, Universidade Federal Fluminense, \\ Campus da Praia Vermelha, \\ Niterói, RJ, 24.210-340, Brazil. \\ Received on 28 February, 2005
}

\begin{abstract}
A one dimensional non-equilibrium stochastic model is proposed where each site of the lattice is occupied by a particle, which may be of type A or B. The time evolution of the model occurs through three processes: autocatalytic generation of $\mathrm{A}$ and $\mathrm{B}$ particles and spontaneous conversion $\mathrm{A} \rightarrow \mathrm{B}$. The two-parameter phase diagram of the model is obtained in one- and two-site mean field approximations, as well as through numerical simulations and exact solution of finite systems extrapolated to the thermodynamic limit. A continuous line of transitions between an active and an absorbing phase is found. This critical line starts at a point where the model is equivalent to the contact process and ends at a point which corresponds to the voter model, where two absorbing states coexist. Thus, the critical line ends at a point where the transition is discontinuous. Estimates of critical exponents are obtained through the simulations and finite-size-scaling extrapolations, and the crossover between universality classes as the voter model transition is approached is studied.
\end{abstract}

\section{INTRODUCTION}

The phase transitions exhibited by stochastic models with absorbing states have attracted much attention in recent years, particularly in order to identify and understand the aspects which determine the universality classes in those models. Most of these models have not been solved exactly, but a variety of approximations allow quite conclusive results regarding their critical properties. Stochastic models are, of course, well fitted for simulations, but closed form approximations and other analytical approaches have also been useful in investigating their behavior [1].

One of the simplest and most studied model of this type is the contact process (CP), which was conceived as a simple model for the spreading of an epidemic and proven to display a continuous transition between the absorbing and an active state, even in one dimension [2]. Actually, it was found that the CP is equivalent to other models such as Schlögl's lattice model for autocatalytic chemical reactions [3] and Reggeon Field Theory (RFT) [4]. The CP belongs to the direct percolation (DP) universality class, together with others models such as the Ziff-Gulari-Barshad model of catalysis [5] and branching and annihilating walks with an odd offspring [6]. The DP conjecture states that all phase transitions between an active and an absorbing state in models with a scalar order parameter, short range interactions and no conservation laws belong to this class [7]. This conjecture was verified in all cases studied so far [8].

Here we study a generalization of the $\mathrm{CP}$, with an additional parameter, so that the $\mathrm{CP}$ transition point becomes a critical line. This model is similar to the model proposed by Hinrichsen [9] in the particular case where his parameter $q$ is set equal to one. Since the symmetry properties of this generalized model are the same of the $\mathrm{CP}$, it is expected that this crit-

\footnotetext{
* Present address: Instituto de Investigaciones Fisicas Universidad Mayor de San Andres Casilla 8635, La Paz, Bolivia
}

ical line should belong to the DP universality class. However, at one point of this line the model is equivalent to the zero temperature Glauber model [10], also called the voter model [11], which displays a spin inversion (or particle-hole) symmetry and therefore belongs to another universality class (the compact directed percolation (CDP) class). Thus the critical line in the phase diagram of the generalized model starts at the $\mathrm{CP}$ model and ends at the voter model, a crossover between the two universality classes being observed.

In section II we define the model and show its equivalence to the $\mathrm{CP}$ and the voter model in the appropriate limits. The phase diagram of the model is obtained in one- and two-site approximations in section III. In section IV, results of simulations are shown which lead to numerical estimates of the critical line and of dynamic critical exponents. Other estimates of the critical line and static critical exponents are obtained through the exact diagonalization of the time evolution operator of the model for finite lattices, extrapolated to the thermodynamic limit using finite size scaling in section V. Final comments and the conclusion may be found in section VI.

\section{DEFINITION OF THE MODEL}

Each one of the $N$ sites of a one-dimensional lattice with periodic boundary conditions are occupied by particles A or B. No holes are allowed. Thus, the state of the system at a given time $t$ is described by the occupation variables $\eta=\left(\eta_{1}, \eta_{2}, \ldots, \eta_{N}\right)$, where $\eta_{i}=0$ or 1 if site $i$ is occupied by particles B or A, respectively.

The time evolution of the system is defined by the following Markovian rules:

1. A site $i$ of the lattice is chosen randomly.

2. If the site is occupied by a particle $\mathrm{B}$, it becomes occupied with a particle $\mathrm{A}$ with a transition rate equal to $p_{a} n_{a} / 2$, where $n_{a}$ is the number of A particles in first neighbor sites of $i$. 


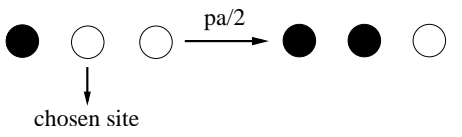

FIG. 1: Autocatalytic creation of a particle A at site $i$. Full circles represent A particles and empty circles denote B particles.

3. If site $i$ is occupied by a particle A, it may become a particle $B$ through two processes

- Spontaneously, with a transition rate $p_{c}$

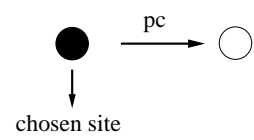

FIG. 2: Example of spontaneous creation of particle B.

- Through an autocatalytic reaction, with a rate $p_{b} n_{b} / 2$, where $n_{b}$ is the number of $\mathrm{B}$ particles in the first neighbors of site $i$.

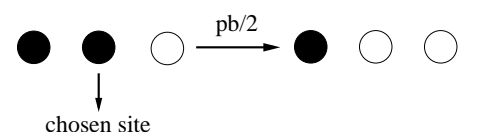

FIG. 3: Example of autocatalytic creation of a particle B.

We define the time step in such a way that the parameters $p_{a}$, $p_{b}$, and $p_{c}$, which are non-negative, obey the normalization $p_{a}+p_{b}+p_{c}=1$, so that only two of them are independent. For convenience, we will discuss the behavior of the model in the $\left(p_{a}, p_{c}\right)$ plane without loss of generality.

The probability $P(\eta, t)$ to find the system in state $\eta$ at time $t$ obeys the master equation

$$
\frac{\partial P(\eta, t)}{\partial t}=\sum_{m}\left\{w_{i}\left(\eta^{i}\right) P\left(\eta^{i}, t\right),-w_{i}(\eta) P(\eta, t)\right\}
$$

where $\eta^{i}$ corresponds to the following configuration

$$
\eta^{i} \equiv\left(\eta_{1}, \ldots, 1-\eta_{i}, \ldots, \eta_{N}\right)
$$

and $w_{i}(\eta)$ is the transition rate of the model, given by

$$
w_{i}(\eta)=\frac{\lambda}{2}\left(1-\gamma \eta_{i}\right) \sum_{\delta} \eta_{i+\delta}+\eta_{i},
$$

where $\lambda=p_{a} /\left(1-p_{a}\right), \gamma=\left(1-p_{c}\right) / p_{a}$, and the sum is over first neighbors of site $i$.

The equation for the time evolution of the mean number of A particles at site $i,\left\langle\eta_{i}\right\rangle$, may be obtained from equations (1) and (3), being given by

$$
\frac{d\left\langle\eta_{i}\right\rangle}{d t}=\frac{\lambda}{2} \sum_{\delta}\left\langle\eta_{i+\delta}\left[1-(2-\gamma) \eta_{i}\right]\right\rangle-\left\langle\eta_{i}\right\rangle
$$

and a homogeneous solution, that is, $\rho \equiv\left\langle\eta_{i}\right\rangle$ and $\phi \equiv$ $\left\langle\eta_{i} \eta_{i+\delta}\right\rangle \forall i$, is given by equation

$$
\frac{d \rho}{d t}=\lambda[\rho-(2-\gamma) \phi]-\rho
$$

In principle, we are not able to solve equation (5) because the function $\phi(t)$ is not known. We may write a differential equation for $\phi(t)$, but in this equation three variable terms such as $\left\langle\eta_{i} \eta_{j} \eta_{k}\right\rangle$ will appear, so that a infinite hierarchy of equations will be obtained. A systematic approximate solution is given below. Although it does not provide precise results, it still furnishes a qualitative picture of the behavior of the system.

This model, similarly to what happens in other out of equilibrium systems, displays absorbing states, which are such that once they are reached, they will never be left. The evolution rules of the model define the state where all sites are occupied by B particles $(\rho=0)$ as absorbing. Besides this stationary state, others may exist such that $\rho=\lim _{t \rightarrow \infty} \rho(t)$ is nonzero. Such states are called active.

It may be useful to remark that this model may be mapped to a spin system if we describe sites occupied by $\mathrm{A}$ and $\mathrm{B}$ particles by an Ising spin variables $\sigma_{i}=1$ and $\sigma_{i}=-1$, respectively. In these variables, the transition rate will be given by

$$
w_{i}^{\uparrow \downarrow}(\sigma)=\frac{\alpha}{2}\left[1+\beta \sigma_{i}-\frac{1}{2}\left(\varepsilon \sigma_{i}+\xi\right) \sum_{\delta} \sigma_{i+\delta}\right],
$$

where $\alpha=\left(p_{a}+p_{b}+2 p_{c}\right) / 2, \beta=\left(p_{a}-p_{b}-2 p_{c}\right) /\left(p a+p_{b}+\right.$ $\left.2 p_{c}\right), \varepsilon=\left(p_{a}+p_{b}\right) /\left(p_{a}+p_{b}+2 p_{c}\right)$, and $\xi=\left(p_{a}-p_{b}\right) /\left(p_{a}+\right.$ $\left.p_{b}+2 p_{c}\right)$.

Finally, this model corresponds to two known models in particular limits. If we make $p_{b}=0$ or $\gamma=1$ the well known contact process is recovered [2],

$$
w_{i}^{(C P)}(\eta)=\frac{\lambda}{2}\left(1-\eta_{i}\right) \sum_{\delta} \eta_{i+\delta}+\eta_{i} .
$$

If now we take $p_{a}=p_{b}$ and $p_{c}=0$ in the spin formulation of the model, the zero temperature linear Glauber model is recovered, also known as the voter model,

$$
w_{i}^{(L G M)}(\sigma)=\frac{\alpha}{2}\left[1-\frac{1}{2} \sigma_{i} \sum_{\delta} \sigma_{i+\delta}\right] .
$$

\section{MEAN FIELD SOLUTION}

An approximate solution of equation (5) is obtained if we write $\phi$ as a function of $\rho$. This approximation does not account for correlations and is known as the one site mean field approximation, assuming $\left\langle\eta_{i} \eta_{j}\right\rangle=\left\langle\eta_{i}\right\rangle\left\langle\eta_{j}\right\rangle$, that is $\phi=\rho^{2}$. Within this approximation, equation (5) may be written as

$$
\frac{d \rho}{d t}=(\lambda-1) \rho-(2-\gamma) \lambda \rho^{2}
$$


and the stationary solution in terms of the parameters $p_{a}$ and $p_{c}$ is

$$
\rho= \begin{cases}0 & \text { if } p_{a} \leq 1 / 2 \\ \frac{2 p_{a}-1}{2 p_{a}+p_{c}-1} & \text { otherwise. }\end{cases}
$$

Therefore, in the one-site approximation the absorbing state is separated from the active state by a continuous transition line located at $p_{a}=1 / 2$ and the behavior of $\rho$ as a function of $p_{a}$ for some values of $p_{c}$ is shown in Fig. (4). As expected,

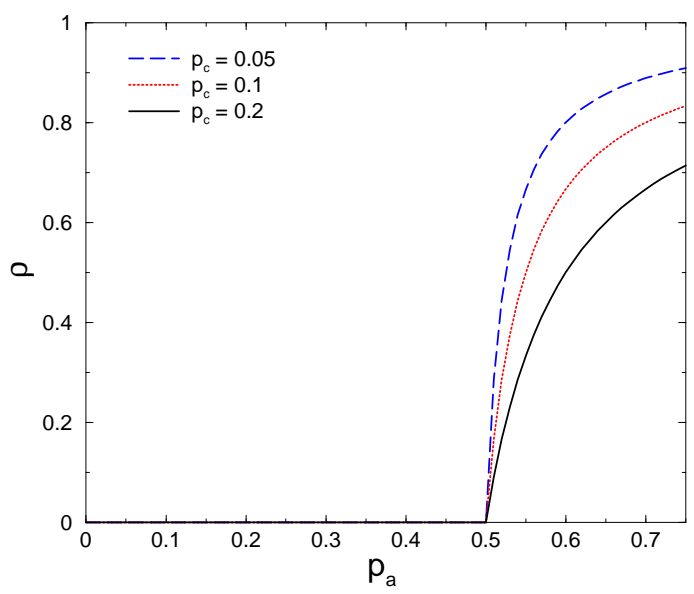

FIG. 4: Density in the steady state as a function of $p_{a}$ for some fixed values of $p_{c}$.

as $p_{c}$ is increased, the density of A sites decreases, since annihilation of A particles is favored. It may be shown that close to the transition line the order parameter behaves as $\rho \sim \Delta^{\beta}$, $\Delta=\left(p_{a}-1 / 2\right)>0$, with $\beta=1$ and, at the critical line, the density decays as $\rho \sim t^{-\varphi}$, with $\varphi=1$. These exponents are identical to the mean field results for the contact process.

A somewhat better approximation is obtained if the set of equations for $\rho$ and $\phi$ is simultaneously solved. As remarked above, an equation for $\phi=\left\langle\eta_{i} \eta_{j}\right\rangle$ shows mean values of products of three $\eta$ variables. Therefore, in order to get a closed set of two equations, these mean values should be approximated as functions of $\phi$ and $\rho$. In doing so, we are neglecting higher order correlations and this is known as the two-site mean field approximation.

The following notation is helpful to obtain the second differential equation of the two-site approximation. We call $\rho=P(\bullet)$, where $P(\bullet)$ is the probability that a site is occupied by an A particle and the probability to find a B particle is $P(\circ)=1-P(\bullet)$. We also define the three probabilities of the configurations of two neighboring sites, and using the relations

$$
\begin{aligned}
& P(\circ)=P(\bullet \circ)+P(\circ \circ), \\
& P(\bullet)=P(\bullet \circ)+P(\bullet \bullet),
\end{aligned}
$$

together with the evolution rules stated above, the following equation for $P(\bullet \circ)$ is found

$$
\begin{aligned}
\frac{d P(\circ \bullet)}{d t} & =\frac{1}{2}\left\{p_{a}[P(\circ \circ \bullet)-P(\bullet \circ)]\right. \\
& \left.+p_{b}[P(\circ \bullet \bullet)-P(\circ \bullet \circ)]\right\} \\
& +p_{c} P(\bullet \bullet)-\frac{1}{2}\left(1+p_{c}\right) P(\circ \bullet) .
\end{aligned}
$$

As already mentioned the probabilities of three site clusters appear in the equation. These will be written in terms of twosite probabilities through the so called pair approximation

$$
P\left(n_{1} n_{2} n_{3}\right) \approx \frac{P\left(n_{1} n_{2}\right) P\left(n_{2} n_{3}\right)}{P\left(n_{2}\right)} .
$$

Within this approximation, a closed system of two equations is found

$$
\begin{aligned}
\frac{d \rho}{d t} & =\left(p_{a}-p_{b}\right) u-p_{c} \rho \\
\frac{d u}{d t} & =\frac{1}{2}\left[p_{a} \frac{r u-u^{2}}{1-\rho}+p_{b} \frac{s u-u^{2}}{\rho}\right] \\
& +p_{c} s-\frac{1}{2}\left(1+p_{c}\right) u,
\end{aligned}
$$

where $u \equiv P(\bullet \circ), r \equiv P(\circ \circ)=1-\rho-u$ and $s \equiv P(\bullet \bullet)=\rho-u$. The first equation above corresponds to equation (5), whereas the second is obtained from equation (13) using the pair approximation (14).

The stationary solutions for the densities are

$$
\rho= \begin{cases}0 & \text { if } p_{a}<p_{a}^{c} \\ \frac{4 p_{a}^{2}-4 p_{a}+p_{a} p_{c}+1-p_{c}}{4 p_{a}^{2}-4 p_{a}+2 p_{a} p_{c}+1-p_{c}} & \text { otherwise, }\end{cases}
$$

where $p_{a}^{c}=\frac{1}{8}\left(4-p_{c}+\sqrt{8 p_{c}+p_{c}^{2}}\right)$. In this approximation the critical value of $p_{a}$ is a function of $p_{c}$. The critical exponents, as expected, have still their mean-field values, only non-universal parameters are different in the two-site approximation as compared to the one-site calculation above. Figure (5) shows $\rho$ as a function of $p_{a}$ for some values of $p_{c}$ in the two-site approximation.

It may be remarked that in the limit $p_{c} \rightarrow 0$ both approximations show a discontinuous transition between two absorbing states, which happens at $p_{a}=1 / 2$. In this limit the model corresponds to a spin model where a given spin is reversed with a probability proportional to the number of spins in first neighbor sites which are in the opposite direction. This might be called a biased voter model when $p_{a}$ is different from $p_{b}$.

\section{SIMULATIONS}

Although, as seen above, the model exhibits an active state in part of the parameter space in the approximate solutions, numerical simulations are useful providing some confidence that this state is not an artifact of the approximation. To our knowledge, for non-equilibrium models an argument similar to the Peierls construction for equilibrium models is still not 


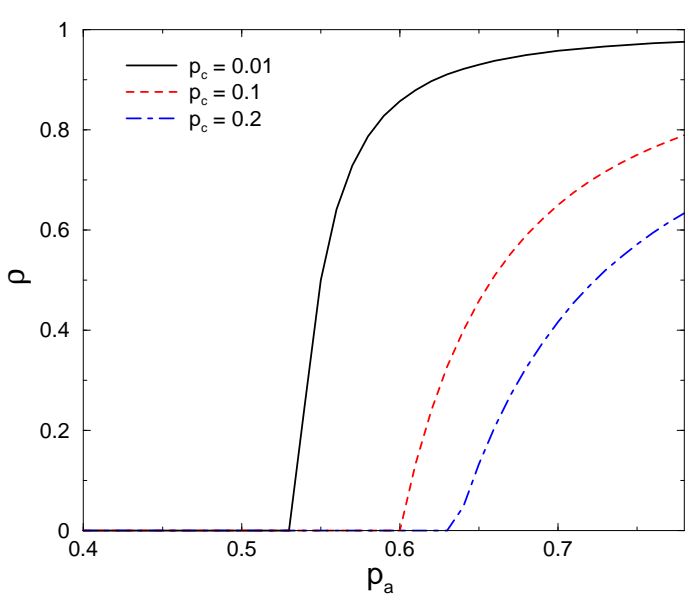

FIG. 5: Density as a function of $p_{a}$ for some values of $p_{c}$, results of the two-site approximation.

available, and therefore no systematic proof of the existence of the active state may be given, although this was accomplished using probabilistic arguments by Harris in his original work on the contact process [2].

The simulation is done for discrete time, and may be described by the following steps:

1. Initially, the system of $N$ sites with periodic boundary conditions is in a state with just one A particle.

2. A list of all sites occupied by A particles is stored, and at each time step one of them is chosen randomly.

3. Once the site is chosen, a random number $p$ uniformly distributed in the interval $[0,1]$ is generated, if $p<p_{a}$ a $B$ particle is replaced by an A particle in one of the first neighbors, if possible. Otherwise, the A particle at the chosen site will be turned into a B particle either through the spontaneous or through the autocatalytic process.

4. To define the process switching $\mathrm{A}$ to $\mathrm{B}$, another random number $q$ is generated. If $q<p_{c} /\left(p_{b}+p_{c}\right)$ the change is spontaneous, otherwise it will happen with a probability proportional to the number of $\mathrm{B}$ particles in first neighbors of the chosen site.

5. The time interval associated with the steps above is $\Delta t=$ $1 / N_{A}$, where $N_{A}$ is the number of the sites occupied by A particles before the step. The process is repeated until either a maximum time $t_{\text {max }}$ is attained or the absorbing state $N_{A}=0$ is reached.

6. Several runs are done and mean values are calculated as a function of time.

The mean number of A particles, $\left\langle N_{A}\right\rangle$, as a function of time for simulations with $N=10000$ sites, $t_{\max }=100000$ and 10000 repetitions is displayed in Fig. (6). The number of sites is sufficiently high to ensure that in the simulations the cluster of A particles is much smaller than the system, thus avoiding finite-size effects in the time interval considered. In a region

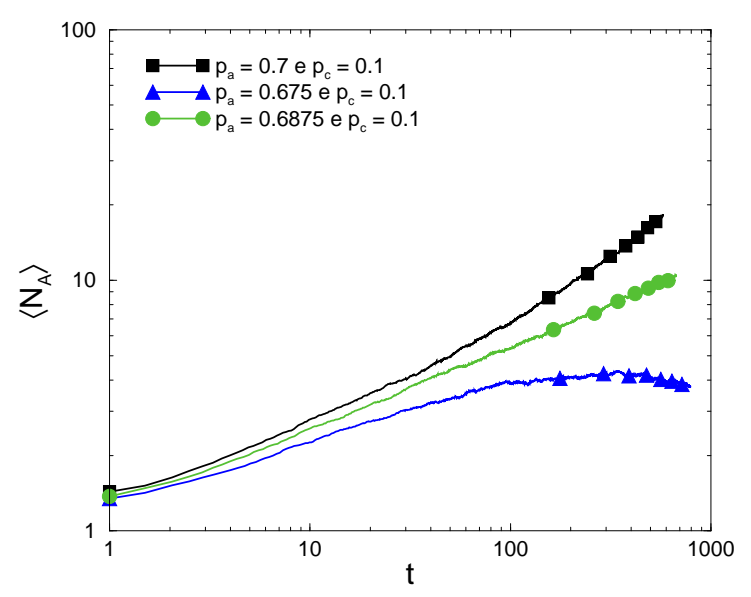

FIG. 6: Results of simulations

of the $\left(p_{a}, p_{c}\right)$ plane an active state is found for long times in the simulations. Three curves are shown in Fig. (6). The convex one corresponds to an active stationary state, while the concave curve signals that the system will reach the absorbing state. These two curves are separated by the third, where a power law $\left\langle N_{A}\right\rangle \sim t^{\theta}$ is seen, corresponding to the critical condition. We adopt, for all critical exponents, the notation proposed in the review article by Hinrichsen [8].

Repeating simulations in the region $\left(p_{a}+p_{c} \leq 1\right)$, the critical line may be estimated. The results of these calculations are shown in Fig. (7).

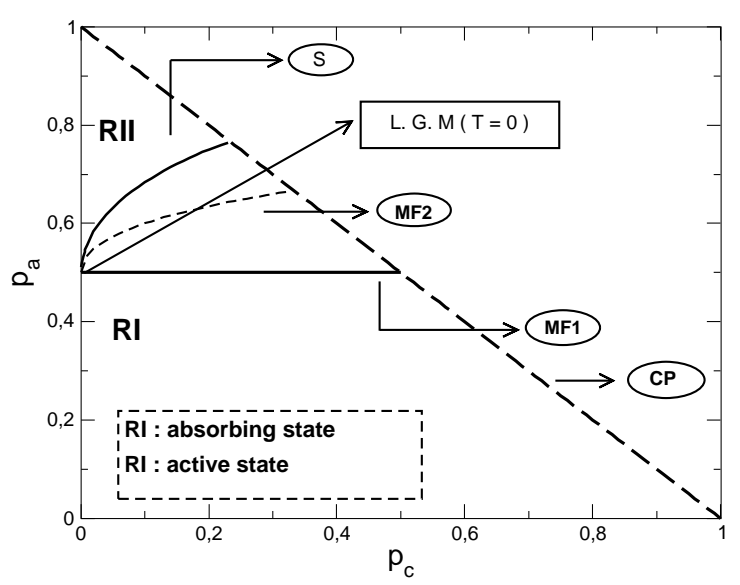

FIG. 7: Phase diagram of the model, showing estimates for the transition line between absorbing and active states in the one (MF1) and two (MF2) sites mean field approximations and simulations (S). In the line $p_{a}+p_{c}=1$ the model corresponds to the contact process (CP) and the unbiased voter model (LGM) is recovered at the point $\left(p_{a}=1 / 2, p_{c}=0\right)$

The estimate for the critical line provided by the simulations display a concavity which agrees with the results of the 
the two-site approximation and, as expected, is situated above the mean field results, which usually overestimate the active region in the parameter space. The value of $\lambda=p_{a} /\left(1-p_{a}\right)$ at the critical line attains the limiting value $\lambda_{c}=3.2945 \pm 0.0116$ as $p_{b} \rightarrow 0$, in good agreement with the estimated critical value for the one-dimensional contact process $\lambda_{c}=3.29785$ (2) [12], as may be appreciated in Fig. (8).

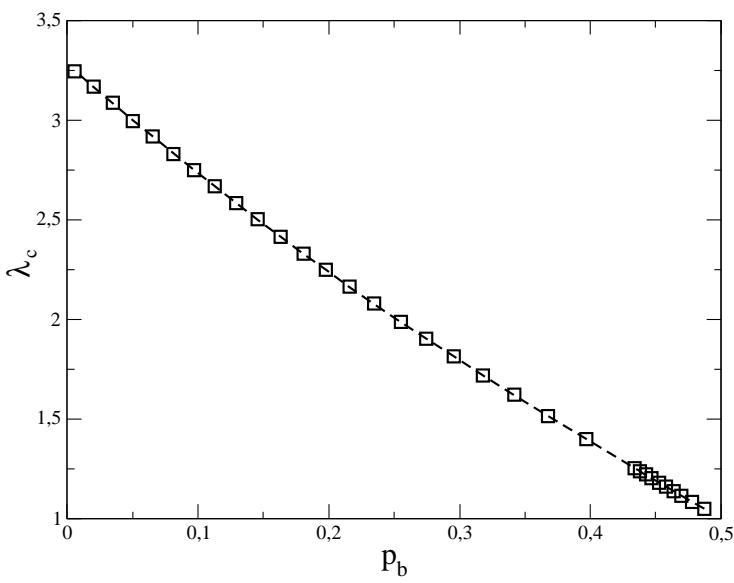

FIG. 8: Critical values of $\lambda=p_{a} /\left(1-p_{a}\right)$ as functions of $p_{b}$. The dashed curve is a fit of a fifth degree polynomial to the points obtained in the simulations.

The simulational result for $\left\langle N_{A}\right\rangle$ as a function of time in the limit of the voter model $\left(p_{a}=1 / 2, p_{c}=0\right)$ is displayed in Fig. (9). One notices that the initial number of particles is almost conserved, showing a narrow dispersion. This aspect

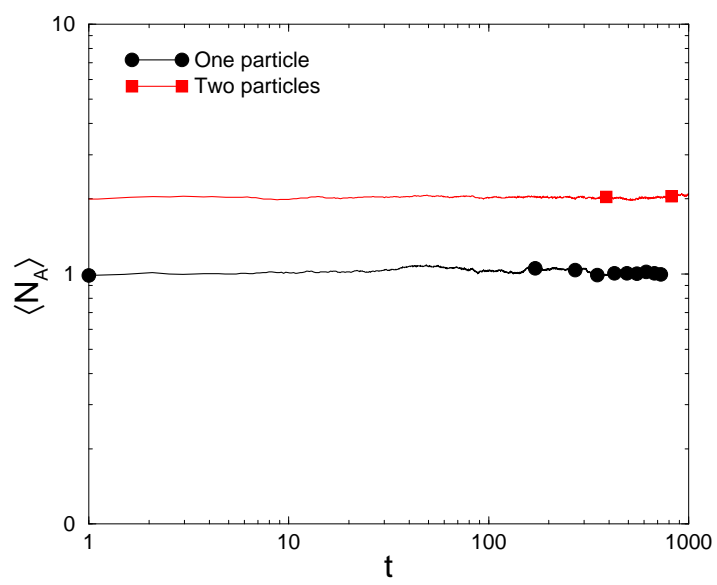

FIG. 9: Simulational results for the mean number of A particles as a function of time for the voter model.

may also be appreciated in the histograms shown in Fig. (10), for $\left(p_{a}=1 / 2, p_{c}=0\right)$ and $\left(p_{a}=0.49, p_{c}=0.001\right)$, with initially one A particle. For the voter model a small dispersion is shown around the maximum value $\left\langle N_{A}\right\rangle=1$, while in the other case the maximum is shifted to the absorbing state $\left\langle N_{A}\right\rangle=0$
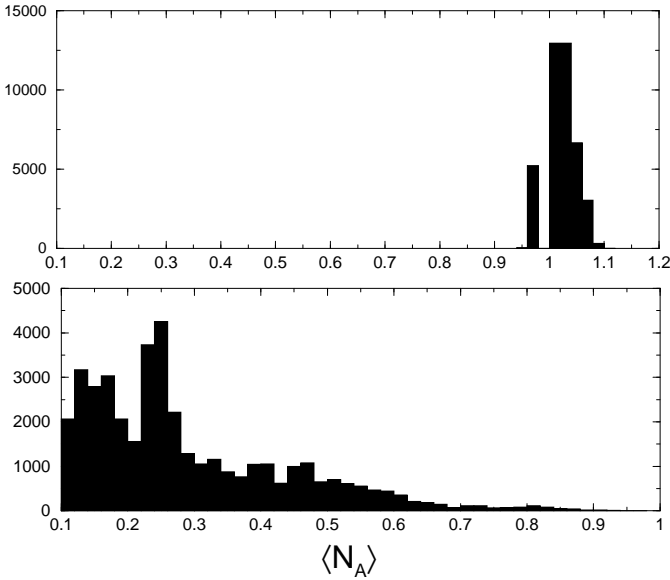

FIG. 10: Histograms of the mean number of A particles. The upper figure corresponds to the voter model $\left(p_{a}=1 / 2, p_{c}=0\right)$ and the other figure is for a point of the parameter space close to the voter model $\left(p_{a}=0.49, p_{c}=0.001\right)$ where the absorbing state $\left\langle N_{A}\right\rangle=0$ is stable.

The simulations allow us to follow the dynamic evolution of mean values that describe the model, such as the mean number of A particles $\left\langle N_{A}(t)\right\rangle$, the probability of survival $P_{S}(t)$ that at the time $t$ at least one particle $\mathrm{A}$ is present in the system and the mean square radius $R^{2}(t)=\left\langle\sum_{i} i^{2} \eta_{i}(t)\right\rangle /\left\langle N_{A}(t)\right\rangle$. These variables satisfy the following scaling forms at the critical point [4]:

$$
\begin{aligned}
\left\langle N_{A}\right\rangle(t) & \sim t^{\theta}, \\
P_{S}(t) & \sim t^{-\delta}, \\
R^{2}(t) & \sim t^{2 / z} .
\end{aligned}
$$

Through scaling and hyperscaling relations, these critical exponents define all other exponents of the model, and thus their values identify the universality class.

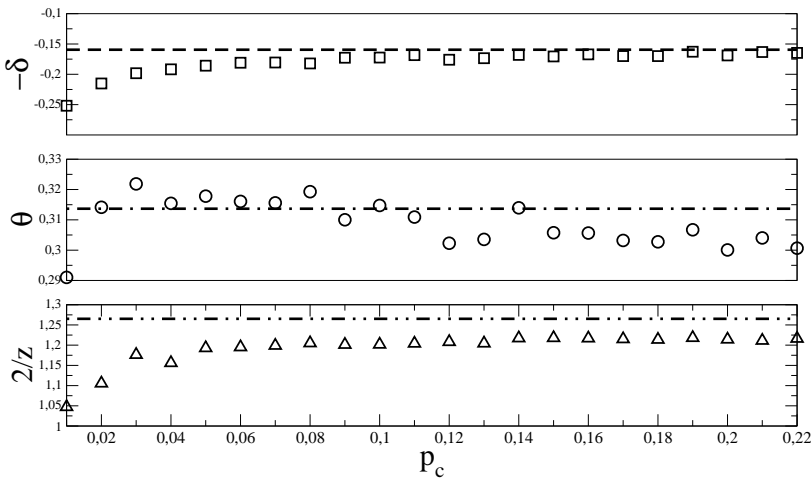

FIG. 11: Dynamical critical exponents as functions of the rate $p_{c}$. The dashed lines indicate the values of these exponents for the CP.

From the simulations, the dynamic critical exponents defined above were estimated as functions of the rate $p_{c}$, as is 
shown in Fig. (11). For most of the values for $p_{c}$, particularly those close to the $\mathrm{CP}\left(p_{c} \approx 0.2327\right)$, the estimated exponents are close to the values known for the CP $(\theta=0.313686$, $\delta=0.159464$, and $2 / z=1.265226$ [13]). The relative errors are displayed in Fig. (12). Also, the hyperscaling relation $2 d / z=4 \delta+2 \theta$ is satisfied numerically with some imprecision, as may be seen in Fig. (13). Some systematic error seems to be present in the estimates for the mean square radius, which propagates to the hyperscaling relation verification. As $p_{c} \rightarrow 0$, however, when the voter model is approached, a departure of all estimates from the $\mathrm{CP}$ values is apparent.
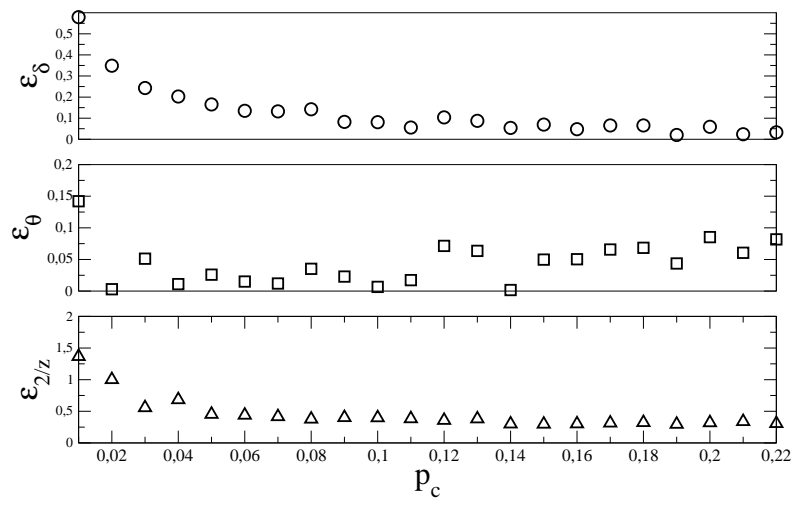

FIG. 12: Relative errors of the estimates of dynamical critical exponents compared to the $\mathrm{CP}$ values.

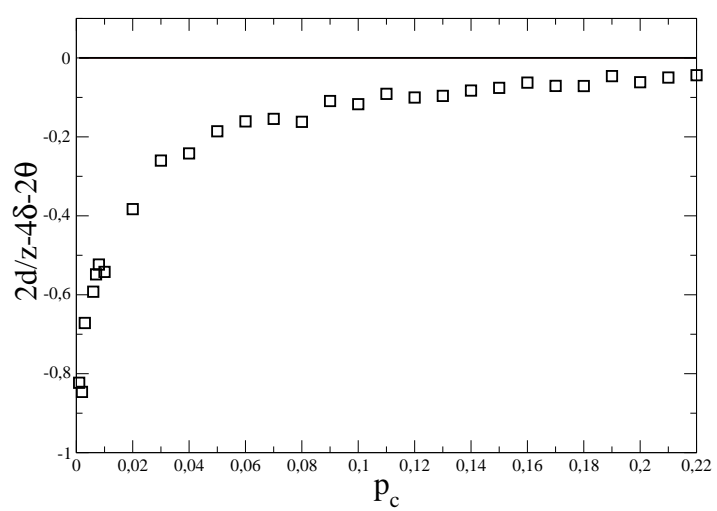

FIG. 13: Verification of the hyperscaling relation for the dynamical critical exponents.

For models with a discontinuous transition with a spin inversion symmetry, such as the voter model, the dynamic critical exponents are given by $\theta_{s}=0, \delta_{s}=1 / 2$ e $2 / z_{s}=1$ and the hyperscaling relation is given by $\delta_{s}+\theta_{s}=d / z_{s}$, corresponding to the compact directed percolation (CDP) universality class [8]. An analysis of the exponents in the limit $p_{c} \rightarrow 0$ may be performed fitting a cubic curve to the estimates, as is shown in Fig. (14) for the exponent $\delta$. The values

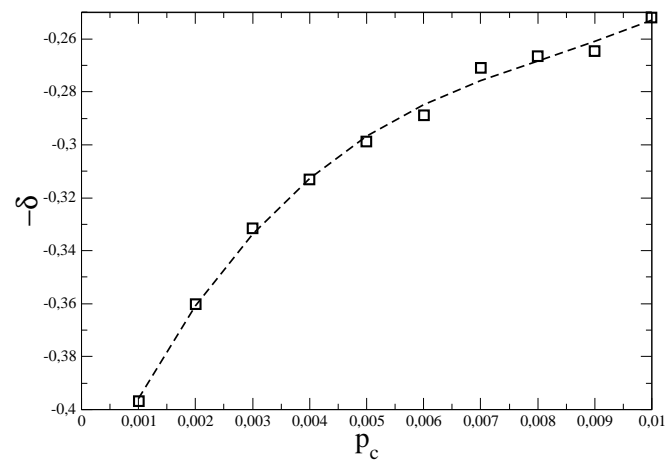

FIG. 14: Limit for $p_{c}=0$ of the dynamical exponent $\delta$.

obtained through this procedure are $\delta=0.44, \theta=0.07$, and $2 / z=1.01$, which are not far from the known values given above. The variation of the estimates for critical exponents with the parameters of the model close to $p_{c}=0$ is probably an apparent effect, due to large fluctuations observed in the simulations in this region, and the whole critical line, with exception of the terminal voter model point, is in the same universality class of the $\mathrm{CP}$, as suggested by the DP conjecture. This situation is quite similar to what is observed in the behavior of the Domany-Kinzel probabilistic cellular automaton (DKPCA) [14], which in a line of its phase diagram corresponds to the $\mathrm{CP}$ with discrete time and parallel update, and also exhibits a crossover from the DP to the compact direct percolation (CDP) universality class [15]. It is believed, although to our knowledge not proven, that changing the update process in a stochastic model may affect non-universal quantities only. The point $p_{a}=1 / 2, p_{c}=0$ may be recognized as a multicritical point, and in its neighborhood any stationary density variable should exhibit the scaling form

$$
g\left(p_{a}-1 / 2, p_{c}\right) \sim(p a-1 / 2)^{e_{g}} F\left(\frac{p_{c}}{\left[p_{a}-1 / 2\right]^{\phi}}\right) .
$$

The critical exponent associated with the density variable $g$, $e_{g}$, should correspond to the CDP universality class, and the scaling function $F(z)$ is singular at a value $z_{0}$ of its argument, which corresponds to the critical line. Thus, the critical line is asymptotically given by $p_{c}=z_{0}\left(p_{a}-1 / 2\right)^{\phi}$ and the amplitude $z_{0}$ and the crossover exponent $\phi$ may be estimated through a fit to the simulational results. Due to the large fluctuations mentioned above, these estimates are not very precise. The values we obtained are $z_{0}=0.47 \pm 0.08$ and $\phi=1.80 \pm 0.03$. In the two site approximation, we found $\phi=2$ both for the model described here and for the DKPCA. Although extensive simulational results for the DKPCA are reported in the literature [16], apparently no estimate for the crossover exponent was obtained from them. 


\section{NUMERICAL DIAGONALIZATION}

Another approach to study $1+1$ dimensional stochastic systems is the exact solution of models with increasing numbers of sites $N$ followed by extrapolations to the thermodynamic limit [8]. This is accomplished writing the master equation (1) as

$$
\frac{\partial}{\partial t}|P(t)\rangle=\hat{\mathcal{S}}|P(t)\rangle
$$

where $\hat{S}$ is the time evolution operator of the model. In the representation in which it is diagonal, vanishing eigenvalues $\mu_{0}=0$ correspond to stationary states of the system. For finite systems with absorbing states, only these states are stationary, and no active stationary state is found.

To study the transition between an active and a stationary state, we may consider the behavior of the eigenvalue with the second smallest absolute value $\Gamma \equiv \mu_{1}$ of the operator $\hat{\mathcal{S}}$. This eigenvalue is related to the quasi-stationary [17] state and will eventually become degenerate with $\mu_{0}$ in the thermodynamic limit, originating the phase transition. Figure (15) shows the behavior of the gap $\Gamma$ for some values of the size $N$. For a

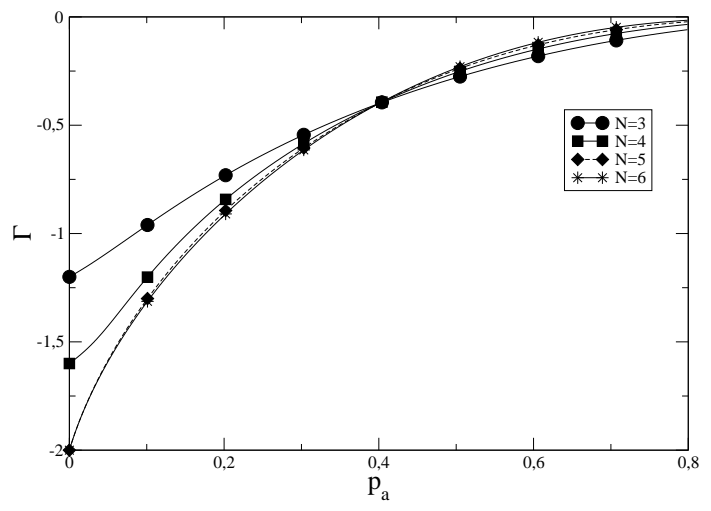

FIG. 15: Gap $\Gamma$ as a function of $p_{a}$ for system sizes $N=3,4,5,6$ with $p_{c}=0.2$.

fixed value of $p_{c}$. the finite size scaling behavior of the gap $\Gamma$ is given by [8]

$$
\Gamma=N^{-z} f\left(\left|p a-p_{a}^{c}\right| N^{1 / v_{\perp}}\right),
$$

where $p_{a}^{c}$ is the critical value of $p_{a}$ for a fixed value of $p_{c}$. Defining the quantity

$$
Y_{N}\left(p_{a}, p_{c}\right)=\frac{\ln \left[\Gamma\left(p_{a}, p_{c} ; N+1\right) / \Gamma\left(p_{a}, p_{c} ; N-1\right)\right]}{\ln [(N+1) /(N-1)]},
$$

we may estimate the critical point $p_{a}^{c}(N)$ finding the intersection of the curves $Y_{N}$ and $Y_{N+1}$ [18]. This procedure resembles the phenomenological renormalization group. The sequence $p_{a}^{c}(N)$ of estimates for a given value of $p_{c}$, with $N=4,5, \ldots M$, was extrapolated to the thermodynamic limit
$N \rightarrow \infty$ using the BST algorithm [19], which even for the limited number of estimates considered lead to rather precise results. Figure (16) shows the extrapolated estimates for the

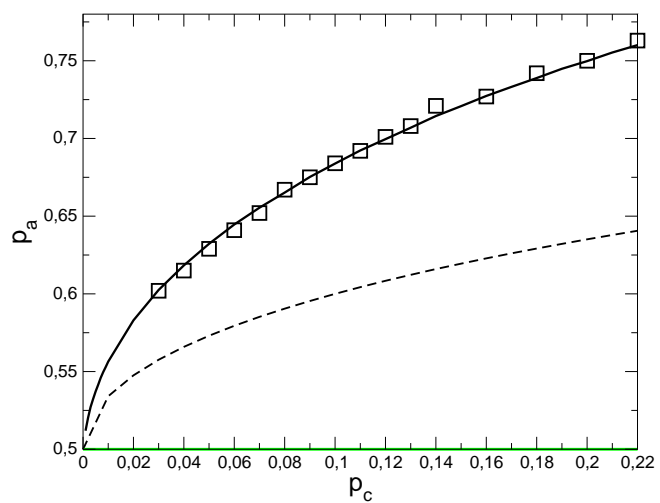

FIG. 16: The squares are the extrapolated estimates obtained from the numerical diagonalization results, compared with the critical lines resulting from simulations (full line) and the two-site approximation (broken line).

critical line, compared with the results provided by the simulations and the two-site approximation results. The agreement between the simulation and the present results is apparent. The results for the critical line close to the CP point $p_{b}=0$ were extrapolated to the $\mathrm{CP}$ limit using a cubic function and the resulting value was $\lambda_{c}=3.3081 \pm 0.0173$, which is in reasonable agreement with a more precise estimate in the literature $\lambda_{c}=3.29785(2)$ [12]. We also estimate the crossover exponent $\phi$, through a fit to the critical line in the neighborhood of the voter model limit and it is results in $\phi=2.24 \pm 0.07$.

Once the extrapolated estimates for the critical line were obtained, the critical exponents $\mathrm{z}$ and $\xi=z-1 / v_{\perp}$ may be estimated using the asymptotic scaling forms

$$
\begin{aligned}
\Gamma\left(p_{a}, p_{c}\right) & \sim L^{-z} \\
\frac{\partial}{\partial p_{a}} \Gamma\left(p_{a}, p_{c}\right) & \sim L^{-\xi},
\end{aligned}
$$

for a fixed value of $p_{c}$ and $p_{a}=p_{a}^{c}$, on the critical line. An estimate of the exponents is obtained for each size of the system, and the estimates are extrapolated to the thermodynamic limit. Our results for these two static exponents are shown in Fig. (17).

Again a departure of the estimates from the CP values may be observed as $p_{c}$ becomes smaller. The CDP values for these exponents are $z_{s}=2$ and $\xi_{s}=1$. Unlike to what was observed in the simulations no systematic trend to these values was observed in the estimates from exact diagonalization as $p_{c} \rightarrow 0$. We noticed, even considering values of $N$ up to 15 , that as $p_{c}$ becomes smaller the convergence properties are poorer, and larger sizes may be necessary to obtain better estimates in this region. The estimates which were obtained from the simulational data seem to be more reliable that the ones which follow from the numerical diagonalization results. 


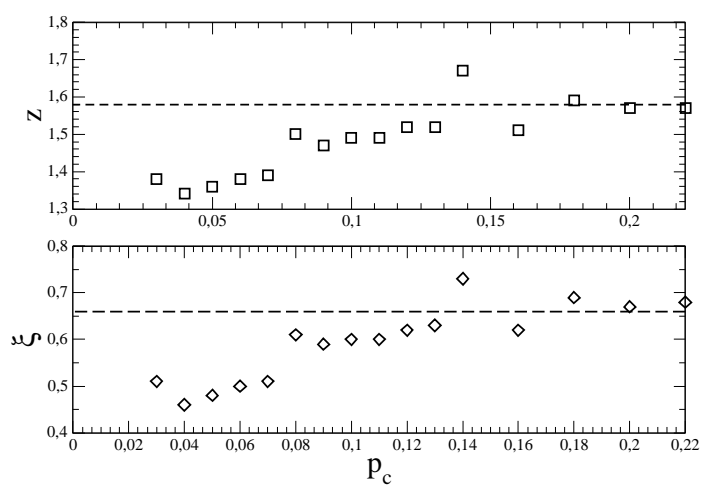

FIG. 17: Static exponents estimated through the exact diagonalization for finite systems. The values for the $\mathrm{CP}$ are shown in dashed lines.

\section{CONCLUSION}

We discussed the transition between an absorbing $(\rho=0)$ and an active $(\rho>0)$ steady states in a stochastic model of interacting particles. This model displays a line of continuous transition between these states starting at a point which corresponds to the contact process and ends at a point where the model is equivalent to the voter model, showing a discontinuous transition between two absorbing states. The phase diagram thus obtained is similar to what is found in a problem of equilibrium polymerization in a grand-canonical ensemble on an anisotropic square lattice [20], where the polymer is modeled as a self-avoiding walk and the activity of a horizontal link is equal to $x$ and a vertical link is associated with an activity $y$. A continuous transition is observed in general in the $(x, y)$ plane, between a non-polymerized $(\rho=0)$ and a polymerized $(\rho>0)$, where $\rho$ corresponds to the fraction of lattice sites visited by the walk. However, at $x=0$ or $y=0$ the walk is one-dimensional, and a discontinuous transition is found between the non-polymerized and the fully polymerized $(\rho=1)$ phases [21].

The localization of the critical line in the parameter space of the model was found in the one- and two-site approximations, as well as through simulations and numerical diagonalization of the time evolution operator. The evidences obtained from estimates of critical exponents suggest that the whole critical line belongs to the DP universality class, and a crossover to the CDP universality class is observed at the terminal voter model point. From the estimates for the location of the critical line close to the voter model point the crossover exponent $\phi=1.80 \pm 0.03$ was obtained. Although the estimated value for $\phi$ is smaller than the mean-field result $\phi=2$, the latter is inside the confidence interval of the estimate, so that it is not possible to decide if the crossover exponent has a nonclassical value. Due to the difficulties we had with the simulations and the numerical diagonalization in the multicritical region, we suspect that another approach is necessary to clear this point. We are presently addressing this point through a series expansion approach. Another extension of this work would be the study of the crossover between the universality class in the Domany-Kinzel cellular automaton, which corresponds to the CP with parallel update in a subspace of its parameter space and also has a point of its phase diagram which corresponds to the voter model.

\section{Acknowledgments}

We thank Prof. Ronald Dickman for many helpful discussions and a critical reading of the manuscript. This research was partially supported by the Brazilian agencies CAPES, CNPq and FAPERJ, particularly through the project PRONEX-CNPq-FAPERJ/171.168-2003.
[1] J. Marro and R. Dickman, Nonequilibrium Phase Transitions in Lattice Models (Cambridge University Press, Cambridge, 1999)

[2] T.E. Harris, Ann. Probab. 2, 969 (1974).

[3] F. Schlögl, Z. Phys. 253, 147 (1972).

[4] P. Grassberger and A. De La Torre, Ann.Phys. 122, 373 (1979).

[5] R. M. Ziff, E. Gulari, and Y. Barshad, Phys. Rev. Lett. 56, 2553 (1986).

[6] H. Takayasu, A. Tretyakov, and A. Yu, Phys. Rev. Lett. 68, 3060 (1992).

[7] H. K. Janssen, Z. Phys. B, 42, 151 (1981); P. Grassberger, Z. Phys. B, 47, 365 (1982).

[8] H. Hinrichsen, Adv. Phys.49, 815 (2000).

[9] H. Hinrichsen, Phys. Rev. E 55.219 (1997).

[10] R. J. Glauber, J. Math. Phys. 4, 294 (1963).

[11] T. M. Ligget, Interacting particle systems, Springer, Berlin
(1985).

[12] R. Dickman and I. Jensen, Phys. Rev. Lett. 67, 2391 (1991); I. Jensen and R. Dickman, J. Stat. Phys. 71, 89 (1993).

[13] I. Jensen, J. Phys A 32, 5233 (1999).

[14] E. Domany and W. Kinzel, Phys. Rev. Lett. 53, 311 (1984); W. Kinzel, Z. Phys. B 58, 229 (1985).

[15] R. Dickman and A. Y. Tretyakov, Phys. Rev. E 52, 3218 (1995).

[16] G. F. Zebende and T. J. P. Penna, J. Stat. Phys. 74, 1273 (1994).

[17] R. Dickman and R. Vidigal, J. Phys. A 35, 1145 (2002).

[18] E. Carlon, M. Henkel and U. Schollwöck, Eur. Phys. J. B 12, 99 (1999).

[19] T.G. Schmaltz et al, J. Phys. A 17, 445 (1984).

[20] J. F. Stilck, Macromol. Symp. 81, 321 (1994).

[21] P. Pfeuty and J. C. Wheeler, Phys. Rev. A. 27, 2178 (1983). 